\title{
The effectiveness of long-term course of Sterimar Mn nasal spray for treatment of the recurrence rates of acute allergic rhinitis in patients with chronic allergic rhinitis
}

This article was published in the following Dove Press journal:

Drug Design, Development and Therapy

\section{Michele Grasso' \\ Marco de Vincentiis' \\ Griselda Agolli' \\ Francesco Cilurzo 2 \\ Raffaele Grasso ${ }^{2}$}

'ENT Department, Head and Neck Surgery, University "Sapienza", Rome, Italy; ${ }^{2} E N T$ Department, Head and Neck Surgery, Lamezia Terme Hospital, Calabria, Italy

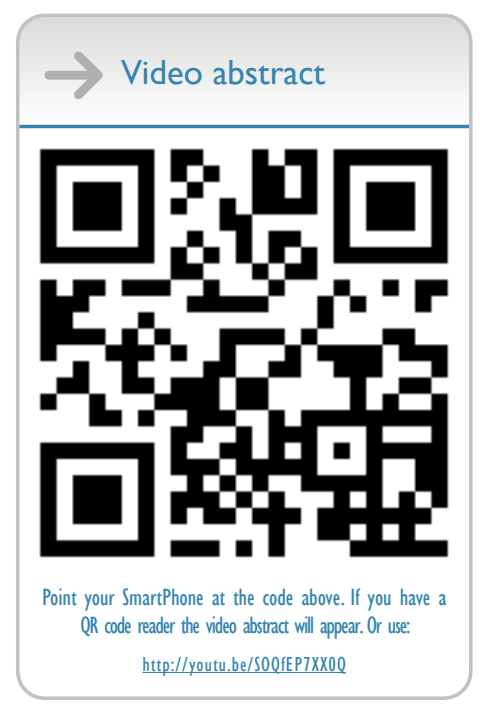

Correspondence: Michele Grasso ENT Department, Head and Neck Surgery, University "Sapienza", Rome, via San Silverio, I5, Rome 00165, Italy Tel +3933346I 6797

Email michelegrasso87@libero.it
Background: The "cost of illness" of the acute allergic rhinitis (AR) episodes in patients with chronic AR is very high in terms of therapy and quality of life. AR represents a worldwide health problem; despite the fact that many standardized treatments have been proposed and used, the recurrence rate of acute rhinitis episodes in springtime is always higher.

Materials and methods: Sixty consecutive patients (13 F and $17 \mathrm{M}$ in group A, $15 \mathrm{~F}$ and $15 \mathrm{M}$ in group $\mathrm{B} ; p=0.60$ ) with chronic AR were enrolled in this prospective, controlled clinical trial. Thirty patients were treated daily for the same 5 months of the following year (2013) with isotonic seawater nasal spray enriched with manganese (Sterimar Mn; 4 puffs/day), whereas 30 patients received only the standard care and were used as control group.

Results: A 5 months course treatment with the nasal Sterimar Mn was able to decrease, significantly ( $p<0.001)$, the number of episodes of acute AR (6.33 episodes in the group of treated patients versus 9.33 episodes in control group). Also, the 5 months quality of life reduced over time (Visual Analogue Scale 5th month 9.90 in treated group versus Visual Analogue Scale 5th month 8.72 in control group: $p<0.001$ ) without the typical adverse effects of the AR standard care therapy.

Conclusion: This study shows the effectiveness of the use of Sterimar Mn for a 5 months therapy in terms of reduction of the number of episodes of acute AR and effectiveness of intrasubject improvement of Visual Analog Scale (quality of life).

Keywords: chronic allergic rhinitis, acute allergic rhinitis, prevention, nasal detersion, Sterimar Mn

\section{Introduction}

Allergic rhinitis (AR) is an inflammatory disease of the nasal mucosa. The typical symptoms are rhinorrhea, sneezing, nasal congestion, nasal pruritus which could extend to eyes, and also redness and lacrimation in many patients. AR can lead to many other consequent diseases including Obstructive Sleep Apnea Syndrome (OSAS) syndrome, rhinosinusitis, asthma, conjunctivitis, and otitis media, and could affect the social life, school outcomes, work production. ${ }^{1} \mathrm{AR}$ is the most common chronic disease in children in USA, one of the most common diseases affecting adults, and the fifth most common chronic disease in the USA overall. It affects nearly one in six US citizens. It impairs the quality of life (QoL), causes loss of school attendance and work, and is responsible for up to $\$ 4$ billion in lost productivity every year. AR generates up to $\$ 5$ billion in direct health expenditures every year. Therefore, there 
are a lot of diagnostic tests and treatments used to manage this disorder, with considerable variation between them. ${ }^{2}$ Therapy is tailored to a patient's symptom burden and QoL and is multimodal. ${ }^{3}$

The Guidelines of Otolaryngology Development Group, AAO-HNSF, recommend intranasal steroids when there is a clinical diagnosis of AR the symptoms of which affect QoL. Oral second-generation antihistamines are recommended when AR is associated with primary complaints of itching and sneezing. A clinical diagnosis of AR should be made when patients present on physical examination and with a history coherent with an allergic base and one or more of the following symptoms: itchy nose, nasal congestion, sneezing, runny nose. Findings of AR coherent with an allergic cause include pale discoloration of the nasal mucosa, red and watery eyes, and clear rhinorrhoea. Physicians should first give empiric therapy. For those who do not respond, when the diagnosis is unclear, and when they need to know the specific causative allergen to target therapy, it is important to perform specific IgE (skin or blood) allergy testing. Physicians should check for the associated conditions such as atopic dermatitis, sleep-disordered breathing, asthma, rhinosinusitis, conjunctivitis, and otitis media. The allergic patients who respond inadequately to drug therapy with or without environmental control measures are candidates for sublingual or subcutaneous immunotherapy. The avoidance of known allergens and environmental controls (ie, the use of air filtration systems, bed covers, removal of pets, acaricides [all the devices targeted to kill dust mites]) are indicated in those who have identified allergens that correlate with clinical symptoms. Surgeons should perform inferior turbinate reduction in patients with AR with nasal airway obstruction and enlarged inferior turbinates with no response to medical management. Patients with perennial, seasonal, or episodic AR can take advantage of intranasal antihistamines. ${ }^{2}$ The recurrence rate of acute episodes of rhinitis in springtime is always higher. These episodes worsen the QoL of the people with chronic AR. According to many authors, ${ }^{4}$ saline nasal irrigation produced a $27 \%$ improvement in nasal symptoms, a $62 \%$ reduction in medicine intake, a $31 \%$ improvement of mucociliary clearance time, and a $27 \%$ increase in QoL. Nasal irrigations using isotonic solution are indicated as complementary therapy in AR. They are well tolerated, inexpensive, easy to use, and there is no evidence showing that a regular, daily saline irrigation adversely affects the patient's health or leads to unexpected side effects. ${ }^{4}$ Manganese is important in the control of allergy, as it manages the release of proinflammatory mediators, such as histamine in the respiratory tract, and has a scavenger activity of promoting superoxide dismutase (SOD).

The aim of this prospective, controlled clinical trial was to understand if a 5 months course of application of isotonic sea water nasal spray enriched with manganese (Sterimar Mn; Laboratori Baldacci, Italy) could be useful to control acute AR attacks in adult patients with chronic AR.

\section{Materials and methods Data collection and study design}

Sixty consecutive patients with chronic AR were enrolled in this prospective, controlled clinical trial. Inclusion criteria were as follows: 1 ) adult patients (18-99 years of age), 2) more than 1 episode of acute AR treated with drugs, such as oral antihistamines, nasal corticosteroids, or nasal decongestants, 3) patients able to give informed consent and complete a scale assessment. Exclusion criteria were the following: 1) presence of asthma, and 2) persistent $\mathrm{AR}(>4$ days/wk, or $>4$ weeks) according to Allergic Rhinitis and its Impact on Asthma (ARIA) guidelines. ${ }^{5}$ To ensure a balance in the samples size groups that results in equal sample, we used a four (4) blocks randomization method. Participants were monitored for seasonal allergy between February and June 2012 (spring time) at the Department of Otorhinolaryngology of the Hospital of Lamezia Terme, Italy. The diagnosis was based on clinical symptoms and on specific IgE (skin or blood) allergy testing. During the same 5 months of the following year (2013), they were randomly divided into 2 groups (Table 1). The first group (A), composed of $13 \mathrm{~F}$ and $17 \mathrm{M}$, was treated with the standard care and named "control group". The second group (B), composed of $15 \mathrm{~F}$ and $15 \mathrm{M}$, was treated with the standard care and Sterimar enriched with Mn (4 puffs/day) for 5 months, and named "treated group" ( $p=0.60)$. The mean age was $39.63 \pm 1.96$ in group A, and $46.63 \pm 1.46$ in group $\mathrm{B}(p=0.11)$. Participants were checked with regard to the following: 1) medication intake and number of episodes of acute AR, 2) examination by the same ear-nose-throat group, 3) self-assessment using a Visual Analog Scale (VAS) score by using a visual scale in which a rating from 1 (poor) to 10 (high) was given for

Table I Patients' features

\begin{tabular}{lll}
\hline $\begin{array}{l}\text { Demographic } \\
\text { indicators }\end{array}$ & $\begin{array}{l}\text { Group A } \\
\text { (standard of care) }\end{array}$ & $\begin{array}{l}\text { Group B } \\
\text { (Sterimar Mn) }\end{array}$ \\
\hline Age & $39.63 \pm 1.96$ & $46.63 \pm 1.46$ \\
Sex & $13 \mathrm{~F}-17 \mathrm{M}$ & $15 \mathrm{~F}-15 \mathrm{M}$ \\
\hline
\end{tabular}

Abbreviations: Sterimar Mn, hysotonic seawater nasal spray enriched with manganese; F, female; $M$, male. 
QoL during the 3rd, 4th, and 5th month of adjuvant treatment. The score changed according to the variation of symptoms of AR, such as sneezing, nasal itching, rhinorrhea, and the degree of satisfaction with adjuvant therapy. During the trial, every acute rhinitis episode was treated with the standard care (antihistaminic, nasal decongestants, or/and corticosteroids) in every patient. Written informed consent was obtained from all study participants.

\section{Outcomes}

Primary endpoint was the ability to decrease the number of episodes of acute AR during the 5 months of observation. The secondary endpoint was the QoL improvement during the 5 months course of treatment.

\section{Analysis of data}

Statistical analysis was performed with SPSS Version 21 (SPSS, Inc., Chicago, IL, USA) for Mac. The comparison of treated group versus controls for sex was performed with Fisher's exact test. The comparison of quantitative variables and scales in the two groups was performed with one-way analysis of variance with bootstrap. All tests were two tailed, and the conventional significance level of 0.05 was adopted.

\section{Ethical considerations}

The research protocol of the study was discussed with and approved by the Ethics Committee of the Hospital of Lamezia Terme.

\section{Results}

The 30 patients (group B) treated with nasal Sterimar Mn showed a significant improvement $(p<0.001)$ in the primary endpoint compared to the 30 untreated patients (group A) (Figure 1). The mean number of episodes of acute AR before therapy was $9.50 \pm 2.64$ in group A and 10.67 \pm 3.14 in group B. A 5 months course treatment with nasal Sterimar Mn was able to decrease, significantly $(p<0.001)$, the number of episodes of acute AR during the 5 months (6.33 episodes in treated patients versus 9.33 episodes in control patients). Also, the 5 months QoL reduced over time (VAS 5th month 9.90 in treated group versus VAS 5th month 8.72 in control group: $p<0.001$ ) without the typical adverse events seen in the AR standard of care. VAS score showed a statistically significant improvement in group B patients versus group A patients even after 3 months, and was maintained up to the 5 th month of treatment $(p<0.001)$ (Figure 2). No side effects to treatment, like epistaxis or discomfort, happened during the 5 months course of the trial. Group B reached a
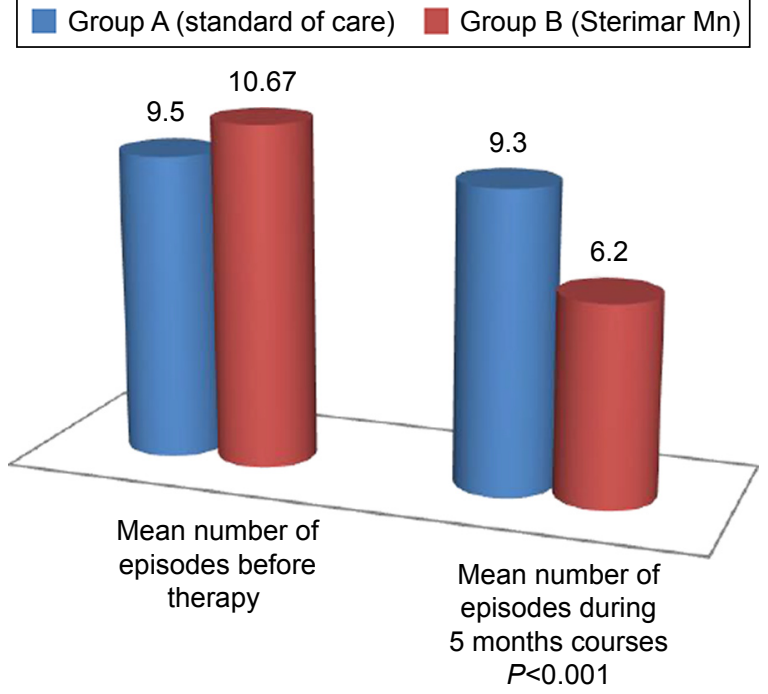

Figure I Mean number of episodes before therapy and during 5 months courses, cross-tabulation.

Notes: Significant differences between treated and control groups; $p<0.00$ I.

significant contribution from the drug on the clinical and subjective point of view.

\section{Discussion}

According to ARIA guidelines, the standard care in the treatment of AR includes antihistamines, nasal decongestants or/ and corticosteroids, cromones, antileucotrienics (if asthma coexists), and specific immunotherapy. ${ }^{5}$ Great importance is given to the removal of allergens and irritants in every step of AR therapy, from those with mild, intermittent to those with moderate-severe, persistent disease. Sterimar Mn decreases the number of episodes of acute AR with a mechanical cleansing, in order to remove antigens, viruses,
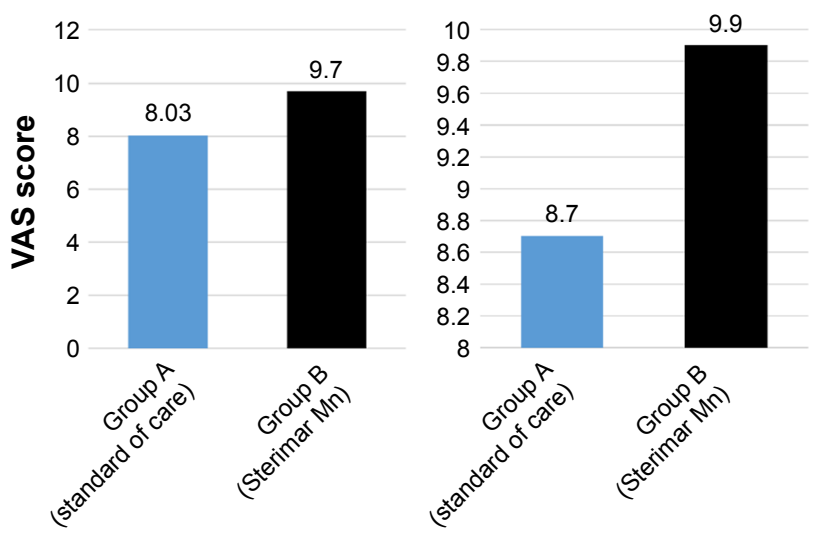

Figure 2 Mean VAS (QoL) during 3 months courses (left side) and Mean VAS (QoL) during 5 months courses (right side).

Notes: The QoL is almost 10,000 after 5 months treatment in the treated group. Significant differences between treated and control; $p<0.00$ I.

Abbreviations: QoL, quality of life; VAS, Visual Analog Scale. 
and bacteria and to improve mucociliary clearance. Results of meta-analysis and systematic review stated that mucosal function improves with a direct physical cleansing. It flushes out debris, allergen, crust, air pollutants, and thick mucus; removes inflammatory mediators; and improves ciliary beat frequency leading to a better mucociliary clearance. Nasal irrigation with saline solution in AR results in the improvement of symptoms, QoL, and mucociliary clearance time. The consumption of antiallergic medication can also be reduced. Nasal irrigation is safe and inexpensive, and represents a nonpharmacological form of treatment. ${ }^{4}$ The neutrophil is considered an important member in the inflammation of the airways in asthmatics. Neutrophils obtained from asthmatic patients generate a threefold increase in stimulated superoxide anion generation when compared to healthy controls. ${ }^{6}$ The mechanism of action of manganese in the control of respiratory allergic phenomena is due to its function as a $\mathrm{Ca}$ channel blocker that prevents the release of proinflammatory mediators such as histamine in the respiratory tract, and owing to its scavenging activity of promoting SOD. Sterimar Mn could reduce acute AR episodes due to the activities of Mn on respiratory mucosa. Excessive production of superoxide anions causes cytokine release, inflammation, formation of chemotactic factors through many pathways such as generation of peroxynitrite, and DNA damage. The mechanism of attenuation of inflammation by SOD mimetics is the reduction of peroxynitrite formation through the elimination of superoxide anions before they react with nitric oxide. Because peroxynitrites are numerous and have proinflammatory and cytotoxic effects, administration of SOD mimetics is clinically very important. ${ }^{7}$

Oxygen radicals are highly chemically reactive substances. They induce bronchoconstriction, increase mucous secretion, and cause microvascular leakage leading to edema formation. Organisms have evolved both enzymatic and nonenzymatic antioxidant protective ways to detoxify bad oxidants. The major intracellular antioxidant enzymes, SOD, catalase, and glutathione peroxidase, inactivate the oxygen radicals, producing a protective result on the airways. Tekin et $\mathrm{al}^{8}$ found significantly lower erythrocyte copper-zinc SOD activity in mild asymptomatic asthmatic patients who had never taken any kind of antiasthma therapy when compared to healthy controls. Manganese SOD and copper-zinc SOD were immunohistochemically highly expressed in the subepithelial glands of nasal mucosa and in the epithelial cells. The olfactory vesicles showed positive immunostaining for manganese SOD and copper-zinc SOD. Epithelial goblet cells and the connective tissue of lamina propria exhibited negative immunostaining for SODs, proving to be vulnerable to oxidative insults implicated in the generation of O2-radicals. ${ }^{9}$ The mechanism of the inhibitory effect of $\mathrm{Mn} 2+$ ions on mast cell secretion is as follows: Mn inhibits the intracellular calcium-stimulated calcium current. This intracellular calcium is mobilized by the effect of inositol triphosphate generated in response to peptides or polyamines or, from pathways that are still being studied, in response to antigenic stimuli. ${ }^{10}$ Variations of intracellular $\mathrm{Ca} 2+$ levels are key features of secretion processes as well as contractile and metabolic occurrences. As strong inhibitors of mast cell secretion, $\mathrm{Mn} 2+$ and $\mathrm{Zn} 2+$ can be accounted for as leaders of a new class of putative antiallergic therapies modulating intracellular $\mathrm{Ca} 2+$ levels. Mn2+ inhibits a calcium-activated calcium flow and thus opposes the reconstitution of intracellular $\mathrm{Ca} 2+$ reserves. ${ }^{10}$ Eosinophils generate large amounts of oxidant species. The eosinophil-dominant type of chronic rhinosinusitis with nasal polyps is related to more extensive disease and a decreased likelihood of surgical success.

SOD is the first-line and only antioxidant enzyme that converts superoxide to hydrogen peroxide. SOD activity in the eosinophilic and noneosinophilic groups was significantly reduced compared to that of the control groups. The reduction in SOD activity and the downregulation of the SOD message seems to be related to eosinophil recruitment and epithelial damage of chronic rhinosinusitis with nasal polyps. ${ }^{11}$ An inappropriate use of the standard of therapy in AR could lead to unlikely side effects, such as rhinitis medicamentosa. ${ }^{12}$ In our study, no side effects were observed during the 5 months course of the trial with hysotonic seawater nasal spray.

VAS is a simple quantitative way that is widely used to enable clinicians to assess the severity of AR and to evaluate treatment efficacy. The VAS can show the variations of symptoms and QoL of patients with AR with high sensitivity. We used the VAS as a simple quantitative tool to assess the burden of AR in primary care. ${ }^{13}$

Studies of nasal irrigations reported different outcomes in the management of AR. ${ }^{14,15}$ According to Nguyen et $\mathrm{al}^{14} \mathrm{a}$ twice-daily nasal irrigation significantly reduced the scores of Mini-Rhino Conjunctivitis Quality of Life Questionnaire at 4 and 8 weeks compared to baseline. In our study, we used Sterimar enriched with manganese (4 puffs/day) for 5 months, in order to achieve significant improvement of symptoms of AR and greater degree of satisfaction of adjuvant therapy compared with the standard therapy alone. A Cochrane review ${ }^{15}$ found no benefit of saline spray over intranasal steroids, and some benefit of saline irrigation $(150 \mathrm{~mL})$ with a hypertonic solution compared to placebo, in patients with chronic rhinosinusitis, because of the different 
bias in the two studies considered. In our study, we treated only patients with chronic AR, comparing the use of adjuvant therapy to nonuse. Since Chong et $\mathrm{a}^{15}$ pointed out the nonactivity of nasal physiological solutions in addition to standard care, it could be that the sum of effects of Sterimar $\mathrm{Mn}$ is responsible for the activity.

\section{Conclusion}

In conclusion, our results demonstrate the effectiveness of Sterimar Mn (4 puffs/day for nostril) for 5 months courses therapy to control the number of episodes of acute AR and to improve QoL. The seawater solution enriched with manganese represents a good tool in the management of the acute AR episode in patients with chronic AR, without the typical side effects seen with common therapy.

\section{Disclosure}

The authors report no conflicts of interest in this work.

\section{References}

1. Feng S, Fan Y, Liang Z, Ma R, Cao W. Concomitant corticosteroid nasal spray plus antihistamine (oral or local spray) for the symptomatic management of allergic rhinitis. Eur Arch Otorhinolaryngol. 2016;273(11): 3477-3486.

2. Seidman MD, Gurgel RK, Lin SY, et al. Clinical practice guideline: allergic rhinitis. Otolaryngol Head Neck Surg. 2015;152(1 Suppl):S1-S43.

3. Kakli HA, Riley TD. Allergic rhinitis. Prim Care. 2016;43(3):465-475.
4. Hermelingmeier KE, Weber RK, Hellmich M, Heubach CP, Mösges R. Nasal irrigation as an adjunctive treatment in allergic rhinitis: a systematic review and meta-analysis. Am J Rhinol Allergy. 2012;26(5): e119-e125.

5. Allergic Rhinitis and its impact on Asthma (Italian Section). Lombardi C, Passalacqua G, et al. http://www.progetto-aria.it/materiale/2017/slidekit-aria-2017.ppt

6. Joseph BZ, Routes JM, Borish L. Activities of superoxide dismutases and NADPH oxidase in neutrophils obtained from asthmatic and normal donors. Inflammation. 1993;17(3):361-370.

7. Li C, Zhou HM. The role of manganese superoxide dismutase in inflammation defense. Enzyme Res. 2011;2011:387176.

8. Tekin D, Sin BA, Mungan D, Misirligil Z, Yavuzer S. The antioxidative defense in asthma. J Asthma. 2000;37(1):59-63.

9. Lai MT, Ohmichi T, Ogawa T, Nishizaki K, Masuda Y. Electron spin resonance spin trapping assay and immunohistochemical localization of superoxide dismutases in the rat nasal mucosa. Acta Otolaryngol. 1997; 117(3):437-446

10. Landry Y, Bronner C, Gies JP, Mousli M. The inhibitory effect of divalent cations on mast cell secretion. Rev Fr Allergol. 1993;33(2): $146-150$.

11. Ono N, Kusunoki T, Miwa M, Hirotsu M, Shiozawa A, Ikeda K. Reduction in superoxide dismutase expression in the epithelial mucosa of eosinophilic chronic rhinosinusitis with nasal polyps. Int Arch Allergy Immunol. 2013;162(2):173-180.

12. Doshi J. Rhinitis medicamentosa: what an otolaryngologist needs to know. Eur Arch Otorhinolaryngol. 2009;266(5):623-625.

13. Demoly P, Bousquet PJ, Mesbah K, Bousquet J, Devillier P. Visual analogue scale in patients treated for allergic rhinitis: an observational prospective study in primary care. Clin Exp Allergy. 2013;43(8): 881-888.

14. Nguyen SA, Psaltis AJ, Schlosser RJ. Isotonic saline nasal irrigation is an effective adjunctive therapy to intranasal corticosteroid spray in allergic rhinitis. Am J Rhinol Allergy. 2014;28(4):308-311.

15. Chong LY, Head K, Hopkins C, et al. Saline irrigation for chronic rhinosinusitis. Cochrane Database Syst Rev. 2016;(4):CD011995.

\section{Publish your work in this journal}

Drug Design, Development and Therapy is an international, peerreviewed open-access journal that spans the spectrum of drug design and development through to clinical applications. Clinical outcomes, patient safety, and programs for the development and effective, safe, and sustained use of medicines are the features of the journal, which has also been accepted for indexing on PubMed Central. The manuscript management system is completely online and includes a very quick and fair peer-review system, which is all easy to use. Visit http://www.dovepress.com/testimonials.php to read real quotes from published authors. 